\title{
A Learning Object Approach for Designing Information Literacy Instructional Materials
}

\author{
Mark Meng \\ Loretto Memorial Library, St. John’s University \\ Staten Island, NY, USA
}

\begin{abstract}
The use of a Learning Object is a new concept in designing online instructional content. It values the creation of small lego-like instructional components (objects) that can be readily assembled, delivered and reused in multiple instructional and learning contexts. Unlike conventional tutorials, learning objects are designed for building a collection of learning resources that are not bound by a particular context or situation. Information literacy instruction lends itself well to the learning objects approach. With this approach, the tagging of lego-like objects allows matching objects with individual competency levels, hence provides greater flexibility and relevancy for end users. Other advantages of developing material to be reused as learning objects such as ease of update and search, customization, interoperability and increased value of content are discussed in relation to information literacy instruction.
\end{abstract}

Currently most online learning materials are designed with a practical objective in mind. Typically, the objective is to create an online course, a training program on mastering certain skills, or a series of exercises for assessing performance competencies. The main purpose of designing such electronic content is to satisfy the immediate demand of a curriculum or the needs of a situation. Usually, the content is not designed to be reused in a different curriculum or situation, nor are they intended to be shared with other instructors or institutions. This electronic content bears all the characteristics of a conventional course taught in a classroom or performance training program in a controlled environment except that they are put online in electronic format for free access. A good example of such electronic learning content can be seen in the Information Literacy Tutorial developed by St. John's University, New York (Figure 1)

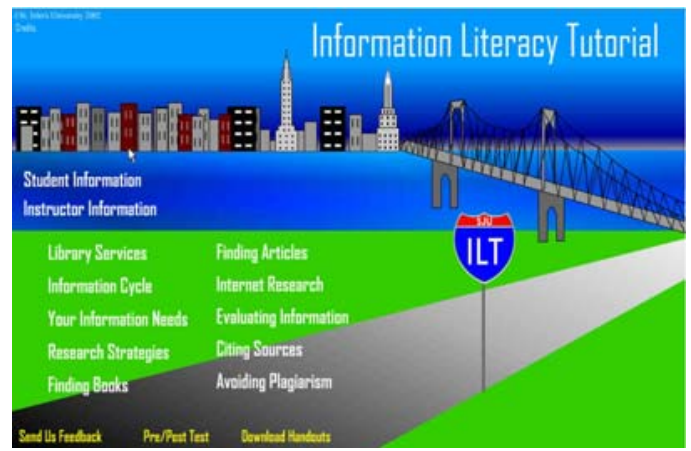

Figure 1 : Information Literacy Tutorial

The advantage of designing online content in this manner seems obvious. First of all, the instructors and designers can retain firm control of all aspects of the content materials to be learnt. They can predetermine the levels of difficulty, the amount of information, and even the duration of each lesson. Secondly, instructors and designers can apply preferred pedagogical methods to present each lesson, and determine the structural partition and sequence of instruction according to a lesson plan. In the above example, even a guideline is provided on how to use the content for instructors and students; a table of contents is clearly laid out. There are additional sections for 
assessment, feedback, and handouts. Lastly a major advantage of this type of online content is that it benefits greatly from the Internet, which guarantees ubiquitous access, information freedom, asynchronistic delivery, and repeatability of learning lessons. Also, this kind of electronic content is not particularly hard to develop because of its intrinsic similarity to the conventional way of teaching and learning. All that is needed is a good technical transplantation of content from paper and chalk to electronic medium plus all the visual and aural gimmicks that come with digital applications.

Conceptually, this approach to content is no different from that of a textbook or lesson plan. Except that a textbook or a classroom session is moved on to a computer screen, there is no fundamental change. Learners are following a prepackaged route to approach a subject that is embedded with logical or pedagogical justifications. Instructors are still dictating the learning and attempting to control the outcomes. The advantages of mimicking classroom or textbook instruction through an electronic medium might make us forget that learning is an individual act and it happens only if the learner has internalized the content and made it meaningful to him/herself alone. In a traditional setting, textbook and classroom presentations are continually complemented and enriched by personal interaction between instructors and students face-to-face through question and answer and many unpredictable deviations and digressions. While in an online learning environment, learners can gain some benefit from the electronic format, they often completely lose control of the content presentation. How to introduce more personal control to make learning content relevant and its structure flexible for each individual learner is a major concern for online learning instructors and designers.

In most online courses and tutorials, the divisions of the content fall into conventional categories. The titles tend to be generalized descriptions that hardly tell what is actually in it. Once the user clicks on a title, a presentation or a text description will start rolling out in an instructional sequence. Students are able to set the pace of moving from one screen to another, but they have no control over what to view, nor can they change the sequence of presentation. Everything is predetermined. Users are treated equally without distinction. Each user is given a complete lesson and has to go from the beginning to the end, regardless of whether the student ha absorbed certain content or will need a different level of content. This may be the main reason why students easily get impatient with online tutorials, because they have to read or browse through content that is not relevant to their needs. Since they cannot raise questions to lead the discussion to a deeper level or more relevant context, they may quickly click through screens without carefully reading them. In this process of browsing sequenced content they tend to throw out the baby with the bath water altogether.

Using the "Finding Books" lesson in the sample information literacy tutorial as an example, its content can include twenty subtopics that could be treated in a variety of ways, at different levels of difficulty and coverage. However, a survey of existing tutorials indicates that there are no lessons on "Finding Books" that have covered all twenty subtopics, nor do these lessons treat the subtopics with equal attention. Some tutorials have only five elements, whereas others cover ten. Of course, this does not mean that a tutorial with fewer subtopics is superficial and not as good as the ones discussing more topics. In the sample information literacy tutorial from St. 
John's University, there are roughly seven subtopics discussed in the "Finding Books" lesson. The selection/omission was based upon considerations of our unique student body, curriculum requirements, pedagogical concerns, and the flow of the content as a whole. So in the end, it's we, the librarian designers, who have decided what and how things are to be learnt. We establish one fixed learning path, seek out the elements to be covered, and prescribe an order for everyone to follow.

In the digital learning environment, information is produced at an astonishing speed and consumed in unprecedented quantity. Online learners typically have higher demand for efficiency and flexibility of instructional content. We need to reevaluate the ways we structure lessons, present the materials, and deliver the content to the individual in front of a computer screen. We cannot simply convert textbooks or classroom practices to online lessons without making conceptual readjustments. We have to build flexibility into the online instructional system so that it will not just make sense to the "universal" student in our mind, but address the needs of individuals. Once learning objectives and outcome requirements are established, we should provide learners with options to choose what they deem to be most important and appropriate to learn, instead of dictating every process and all content. This is where a learning objects approach may play an important role in designing online instructional systems.

Learning objects is a relatively new instructional technology concept. It is defined as "any digital resources that can be reused to support learning." (Wiley, 7) Those resources usually are "stand-alone pieces of information that are reusable in multiple contexts depending on the needs of the individual user." (Hodgins, 1999) The fundamental idea behind the concept is that all course or lesson content can be broken down into numerous constituent parts and each part should be designed to be relatively independent from other parts as an instructional unit.

A good example to illustrate it would be the "Finding Books" lesson referred to earlier. It can have around twenty individual parts on the topic. In developing instructional materials, designers should make each of these components stand alone like Lego pieces or wood blocks (ji mu.) Lego-like has been widely used as a metaphor for learning objects. However, a newer metaphor is emerging that likens learning objects to atoms in order to avoid the connotation of mechanistic assemblage of childish toys.(Wiley, 20) The purpose should be to develop component objects individually to form an object-base. Then for a given learning or instructional objective, the users, students as well as instructors, can make their own decisions and select components from the object-base to use or form an instructional lesson according to individual needs. Designers should not try to select the components deemed important and omit the parts regarded as less relevant, nor should they introduce relationships among components or sequence the order of approaching them. In short, the design goal should not aim to develop a course or a lesson with a fixed structure and fixed numbers of components.

Ideal learning objects will have only one learning objective for each object. They are free-standing and transportable among applications and environments. They will be non-sequential and can be adapted to audiences beyond the original target audience. They have a clear metadata schema for easy searching and identification. (Longmire 2000) 
There are many advantages in building small blocks of instructional units. First they are very flexible. Since each object has only one objective, it can be fairly small. As a result, it is easier to design, and much easier to change and update. Because of their small size, they can be designed to be independent of a particular context; hence it is much easier to reuse them in a variety of different situations. For example, it would be much harder to take one or two parts out of the lesson "Finding Books" or add one or two additional parts to it. This is because when we set out to design it, we planned it as a complete lesson that aims specifically for the average student in a particular group. With a learning object approach, designers must enlist a sort of "double vision" that requires them to design the content not only as a course for a particular context but also as stand-alone information at the desired level of granularity. (Longmire 2000) If objects are designed in this manner, they can be easily reused or reassembled for other situations. Instructors can recontextualize objects for a given instructional goal.

If all the related elements are designed independently for equal access and selection, they can also be easily customized for individual users. Users don't have to follow a fixed sequence of approaching a lesson. They can choose only the parts they want to learn or need to learn and not even touch the parts they already know or they don't need to learn. Through a well-designed and stocked objects base, students will even be able to select objects with different formats, styles, and levels of difficulty to personalize the learning to their own preference.

Scholars and instructional designers have also recognized that a learning management system in which content is designed as reusable learning objects and stored in an object base for easy access and retrieval will greatly facilitate competency-based learning. (Wagner, Tuso 2000) Competency is usually defined as a combination of skills, abilities, and knowledge needed to perform a specific task. Learning that focuses on achieving a set of competencies emphasizes one should only learn what one needs to learn, not what a course designer thought one should learn. A learning plan should address only the knowledge or performance gaps, so the learner doesn't have to revisit what he/she already knows or learn more than he/she needs for a given performance goal. For example, if the only thing a student doesn't know in all twenty topics in the "Finding Books" lesson is Boolean logic, then he/she should only learn the part on Boolean logic instead of repeating other topics in the lesson. The challenge for implementing competency-based learning is the lack of appropriate content that is sufficiently modular and granular to be truly adaptive and effective when applied to a learning community with diverse levels of competencies. Most existing content is still in the form of courses or fixed lessons exemplified in the Information Literacy Tutorial in Figure 1. However, with learning objects, the smaller instructional units and the tagging of Lego-like or atom-like objects allows instructional units to be accessed independently to meet individual learning needs and performance gaps. It will be able to provide the Just-in-time, Just-enough, and Justfor-me instruction that many online learners are seeking.

Information literacy instruction lends itself very well to the learning objects approach. By nature, the content of information literacy is predominantly skills-based and abilities-based instead of knowledge-based. The ACRL Information Literacy Competency Standards for Higher Education sets very clear performance indicators 
for all five standards required for an information literate person. With such welldefined criteria in place, it is relatively easy to design the instructional content as learning objects, each targeting one of the specific indicators.

A prototype website for collecting and delivering instructional materials based on the learning object concept could be laid out like that of Figure 2. This Learning Objects Repository: Information Literacy is just a framework and is yet to be refined and stocked with content. For the purpose of getting the message through, the term "object" is deliberately used in titles for several sections in the page. In reality it can be readily substituted with terms like "resources," "unit," "modules," and "instructional materials." The layout of the page is very simple but doesn't necessarily have to be so. Unlike most online courses and tutorials with well structured table of content and categories occupying the main section of the home page, the Learning Objects Repository has a search box dominating the main viewing section of the repository. This might be regarded as a minor layout design issue, but it should be one of the most important features for a learning objects repository. The search box empowers users and lets them take control of their own learning. Since all objects are designed to be modular and granular and are labeled with metadata tags, it is possible for learners to search for objects with high a degree of precision. The objects in the repository should be free-standing instructional units devoted to only one single learning objective. For example, for the twenty components of Finding Books, there will be twenty different instructional objects. The objects can be in a variety of formats and types. They could be power point presentations, video clips, sound recordings, animations, demos, exercises, tests, games, or even just text files as long as they fulfill the desired instructional objective.

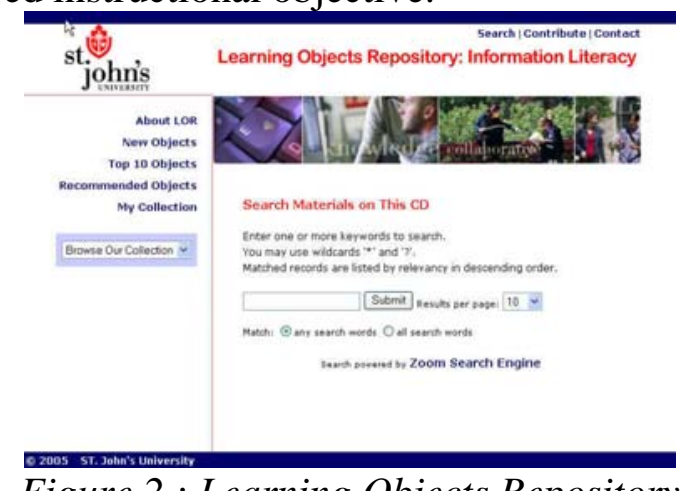

Figure 2 : Learning Objects Repository

Applying a learning object concept in designing a learning system does not exclude the intervention of instructors. The features of a conventional approach can be retained to provide added value to a learning objects repository. On the left side of the page are categorized content sections to add more value to the page and the space. Under the "Browse the Collection" section, a drop-down menu will list links to pages that classify objects according to lessons, topics, formats, types of materials, structured exactly like most online courses and tutorials. Instructional designers and educators can still predetermine the course of study and allow users to select and use well defined and structured lessons if appropriate. However, the lessons will differ from the conventional course as the lessons in the repository are assembled together by using learning objects in the object base. They can be easily disassembled and then reshuffled to form new lessons for different learning goals or contexts. 
Learning object is still a novel concept and there remain many challenges. In order to successfully and efficiently implement the approach it requires thoughtful planning. The first step towards a successful design is to clearly articulate the specifications for developing learning materials. This will include decisions and guidelines for technologies to be used, metadata structures, levels of granularity, editorial control, and document templates. With well defined specifications and a sound delivery platform, a learning objects system not only can deliver flexible and personalized content for learners, but can also stimulate and facilitate a dynamic exchange and growth of learning content for all members of educational community. 


\section{References}

Hodgins, W. (1999). Learning objects: status and direction. Retrieved March 20, 2003, from www.learnativity.com

Longmire, W. (2000). A primer on learning objects. Retrieved October 10, 2003, from www.learningcircuits.org.

Tuso, G. \& W. Longmire (2000). Competency-based systems and the delivery of learning content. Learning Without Limits, vol. .3 Retrieved January 28, 2005, from http://www.learnativity.com/download/LwoL3.pdf

Wagner, E. (2000). E-Learning: Where cognitive strategies, knowledge management, and information technology converge. Retrieved January 28, 2005 from http://www.learnativity.com/download/LwoL3.pdf

Wiley, D. (2002) Connecting learning objects to instructional design theory: A definition, a metaphor, and a taxonomy. The Instructional Use of Learning Objects - Online Version. Retrieved October 20, 2004 from http://www.reusability.org/read 
Reproduced with permission of the copyright owner. Further reproduction prohibited without permission. 